CARNETS DE Carnets de géographes

GÉOGRAPHES.

$7 \mid 2014$

Les espaces de l'entre-deux

\title{
Montagnes frontalières des innovations à la marge
}

L'exemple des Alpes occidentales

\section{Lauranne Jacob}

\section{OpenEdition}

Journals

Édition électronique

URL : http://journals.openedition.org/cdg/403

DOI : $10.4000 /$ cdg. 403

ISSN : 2107-7266

Éditeur

UMR 245 - CESSMA

\section{Référence électronique}

Lauranne Jacob, « Montagnes frontalières des innovations à la marge », Carnets de géographes [En

ligne], 7 | 2014, mis en ligne le 01 décembre 2014, consulté le 19 avril 2019. URL : http://

journals.openedition.org/cdg/403 ; DOI : 10.4000/cdg.403

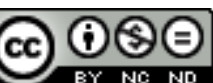

La revue Carnets de géographes est mise à disposition selon les termes de la Licence Creative Commons Attribution - Pas d'Utilisation Commerciale - Pas de Modification 4.0 International. 


\title{
MONTAGNES FRONTALIERES DES INNOVATIONS A LA MARGE \\ L'exemple des Alpes occidentales
}

Lauranne JACOB

Uniı ersité de Grenoble-Alpes et Uniı ersité de Genèı e Labex ITEM $^{1}$

PACTE - CERDHAP

Département de géographie et enı ironnement de l'UNIGE laurannejacob@hotmail.fr

\begin{abstract}
Résumé :
Les montagnes frontalières peuı ent être considérées à double titre comme des espaces d'entre-deux; d'abord par leur localisation géographique à la limite de deux territoires nationaux, ensuite par leurs caractéristiques rurales et spécifiques. Cette situation d'entredeux, plutôt qu'un handicap, se réı èle être daı antage un atout. En effet, les populations confrontées à des contraintes inédites et très particulières, n'ont d'autre alternatiı e que d'innol er aı ec les spécificités de leur territoire et les outils et moyens offerts par les politiques nationales et européennes. La discontinuité légale que représente la frontière est renforcée en montagne par l'obstacle que représente le relief. Cette situation inédite a des conséquences spécifiques et implique une attention particulière lorsqu'on étudie les coopérations transfrontalières qui se déı eloppent sur ces territoires. Cet article étudie la place de ces espaces d'entre-deux dans l'Europe de la cohésion.
\end{abstract}

\begin{abstract}
:
Mountain borders can be considered as "in-between spaces" for two reasons: at first, because they are localised on a border of two national territories and secondly because they hal e rural and specific characteristics. This « in-betweenness » is finally more an asset than a disability.

Indeed, populations faced with new and highly specific constraints don't hale other alternatil es than to innol ate with the strengths of their territory and the tools and resources offered by national and European policies. The border is a legal discontinuity,

\footnotetext{
${ }^{1}$ « Ce tral ail a bénéficié d'une aide de l'Etat gérée par l'agence nationale de la Recherche au titre du programme « inı estissements d'aı enir » Labex ITEM-ANR-10-LABX-50-01 »
} 
which is strengthened by the mountain barrier. This new situation has specific consequences and inı olı es special attention when considering mountain cross-border cooperation. This paper examines the role of "in-between spaces" in the Europe's cohesion. 
Les marges peuı ent être considérées comme des espaces d'entre-deux du fait de leurs caractéristiques hybrides. Souı ent enı isagées uniquement dans un rapport centrepériphérie, leur étude se réı èle biaisée et ne liı re pas tout son potentiel. $A$ la fois témoins des transformations issues du centre, ce sont aussi et surtout des lieux de dépassement, de transgression et d'inno ation où l'altérité est le concept fondamental pour comprendre les liens qui se tissent au jour le jour dans un cadre plus ou moins formel et formalisé.

Nous proposons de traiter le cas des montagnes frontalières, que nous considérons à la fois comme des barrières et des carrefours. Ces deux notions, couramment utilisées pour décrire les montagnes du monde, se réı èlent renforcées par la présence de frontières étatiques. L'objectif de cet article est de démontrer que les montagnes frontalières, bien qu'en situation de marginalité spatiale, tirent partie de leurs spécificités et sont en mesure d'innol er.

Notre propos se concentrera sur les coopérations transfrontalières montagnardes alpines, plus spécifiquement celles situées sur les frontières franco-italienne et franco-italo-suisse. Nous illustrerons notre réflexion par des exemples tirés de recherches en cours sur les coopérations transfrontalières alpines, plus précisément aı ec le cas de l'Espace Mont-Blanc: coopération transfrontalière tri-nationale autour du massif du Mont-Blanc (France, Italie, Suisse) et celui de la coopération transfrontalière entre deux Parcs : le Parco Naturale delle Alpi-Marittime (en Italie) et le Parc National du Mercantour (en France). Ces recherches sont menées selon une approche pluridisciplinaire principalement géographique et juridique, mais faisant aussi appel à la sociologie et l'anthropologie.

Après aı oir démontré comment la montagne et la frontière produisent de "l'entre-deux ", nous mobiliserons les concepts de "liminalité » ou "liminarité » et " d'hybridation " pour les étudier. Enfin nous décrirons les modalités d'innol ation des coopérations transfrontalières en matière de gou ernance, à traı ers l'appropriation d'outils juridiques nouı eaux issus de l'Union Européenne.

\section{Frontières montagnardes, marges ou confins dans l'Europe de la cohésion territoriale et de la compétitivité...}

Les espaces de coopération transfrontalière de montagne sont à double titre des territoires marginaux. Tout d'abord, ils sont localisés géographiquement aux confins des territoires nationaux et se trouı ent, par leurs spécificités morphologiques et climatiques, isolés par rapports aux centres urbains. Etymologiquement, le mot « marge » renı oie au bord, à une extrémité, la frontière extrême limite de la souı eraineté étatique, tandis que le terme " confins " renı oie à une limite qui existe par rapport à une discontinuité (Prost, 2004). Ces deux termes s'appliquent parfaitement à notre objet; néanmoins le ı ocable de marge nous paraissant ou rir daı antage de perspectiı es, nous le priı ilégierons donc. Ils peuı ent aussi être qualifiés d'espaces d'entre-deux. En effet, le territoire qui se dessine progressiı ement autour de la frontière, selon un long processus de recomposition territoriale, présente des caractéristiques propres liées à la confrontation de deux systèmes juridiques, normatifs parfois très différents comme c'est le cas al ec la France, Etat fortement centralisé et ses I oisins suisse et italien dont les régions et cantons bénéficient d'une plus grande autonomie.

\section{Défonctionnalisation de la frontière et entre-deux}


"Les frontières sont des entraves artificielles au développement et leur suppression constitue, de même que la promotion de liaisons transnationales et interrégionales, un objectif de longue date de la politique de cohésion. "Cette citation est tirée du cinquième rapport de la Commission européenne sur la cohésion intitulé « Promouı oir la compétiti ité et la conı ergence ${ }^{2}$. Nous ne pouı ons que nous étonner de la formulation maladroite de la Commission. $S^{\prime}$ il est tout à fait exact que les frontières sont des limites politiques artificielles plus ou moins durables, dire qu'elles sont des entraı es au déı eloppement c'est faire fi du leı ier de déı eloppement économique qu'elles représentent dans les zones transfrontalières. Obstacles, les frontières le sont indéniablement; toutefois le mot " entraı e " nous paraît malencontreux, tout comme l'objectif de suppression des frontières de la politique de cohésion. La suppression des frontières ne peut-être le fait d'une politique de cohésion, car elle ébranlerait la souı eraineté territoriale de tous les Etats frontaliers dont le territoire ne serait plus borné par des frontières. Or ce critère est incontournable pour définir ce qu'est un Etat. Plutôt que de parler de suppression des frontières, il nous paraît plus juste de remplacer cette formulation par le concept de "défonctionnalisation " des frontières. Claude Raffestin attribue à la frontière trois fonctions principales : légale, fiscale et de contrôle. Nous pensons que le degré d'intensité de ces trois fonctions est I ariable au cours du temps et influe directement sur la porosité de la frontière, autrement dit sur sa capacité à faire limite ${ }^{3}$. La catégorie générale "limite" englobe la notion de frontière. Cette défonctionnalisation est directement responsable de l'effacement des frontières et a des conséquences directes sur le processus de territorialisation autour de la frontière. Dans le contexte actuel d'européanisation, les fonctions fiscale et de contrôle sont fortement déı aluées, en particulier la seconde pour ce qui est des frontières internes à l'Union européenne, tandis que la fonction normatil e tend à rester constante au cours du temps du fait du maintien de la souı eraineté des Etats membres de l'Union. Ainsi, l'intensité de la limite est donc sérieusement affaiblie, les relations transfrontalières se dés eloppent d'autant plus facilement que les frontières sont ouı ertes et que les contrôles douaniers sont restreints en application des Accords de Schengen sur la libre circulation des personnes, des biens et des serı ices. La présence d'une frontière ou erte induit aussi une différenciation, encore nommée " effet-frontière " qui fait émerger de noul eaux rapports au territoire (Amilhat-Szary et Fourny, 2006). Les populations frontalières jouent sur les différentiels qui se créent et tirent profit des aı antages comparatifs plus ou moins fluctuants dans le temps, principalement en matière de fiscalité. Claude Raffestin (Raffestin, 1974) décrit aussi la frontière comme pro oquant un effet de disjonction entre deux espaces sociaux ayant des effets positifs et négatifs dans l'espace géographique autour de la frontière, c'est en ce sens que l'on peut parler d'espace d'entre-deux. En montagne, la fermeture ou inı ersement l'our erture de la frontière a des conséquences sur l'effet barrière de la montagne, celui-ci s'en trouı e grandement renforcé en cas de fermeture (Sacareau, 2003). Progressiı ement un

${ }^{2}$ Cinquième rapport sur la cohésion économique, sociale et territoriale: "Inı esting in Europe's future" http://ec.europa.eu/regional_policy/sources/docoffic/official/reports/cohesion5/index_fr.cf $\underline{m}$

${ }^{3}$ Communication orale DREVON G., JACOB L. " Limites et frontières", Séminaire Méthéogéo, Le Pradel, 27 juin 2013 
espace d'entre-deux se forme autour de la frontière. Ainsi, ce territoire d'entre-deux emprunte des caractéristiques aux deux espaces séparés par la frontière. Les habitants des régions frontalières parlent plusieurs langues, utilisent plusieurs monnaies, adaptent leurs habitudes en fonction des al antages de chaque territoire.

Cet « effet frontière " n'est que potentiel, le territoire en fonction de ses spécificités ne se trouı e pas toujours en mesure d'exploiter ces différentiels. De ce fait, on note un écart important entre les régions métropolitaines telles que Genèle et les coopérations transfrontalières alpines étudiées. Par exemple, les flux de frontaliers sont moindres par rapport à ceux constatés dans les régions métropolitaines transfrontalières comme à Genèı e pour rester dans les Alpes. De plus, la population résidant dans les périmètres de coopération est très faible, autour de 100000 habitants pour l'Espace Mont-Blanc. Concrètement, peu de réels flux de traı ailleurs d'un pays à l'autre peuı ent être obserı és, quantifiés et analysés du fait du manque de données transfrontalières, notamment statistiques. Les régions frontalières montagnardes sont sou ent dépourı ues d'obserı atoires comme il en existe sur de nombreuses frontières. En reı anche, les échanges commerciaux sont importants et facilement quantifiables, cependant le commerce international s'est dél eloppé indépendamment des relations de coopérations transfrontalières. II est donc compliqué de décrire précisément les habitudes de ı ie des populations résidant dans un périmètre restreint autour de la frontière. L'Espace Mont-Blanc, coopération transfrontalière autour du massif du Mont-Blanc entre la France, l'Italie et la Suisse, s'est doté depuis 2013 d'un obserı atoire, comprenant des indicateurs qui fonctionnent sur la base du modèle DPSIR (Forces - Pressions - Etat - Impacts - Réponses) ainsi que d'un géonaı igateur ${ }^{4}$. II existe en rel anche, des programmes tels que Alpinspace (Espace Alpin) ou encore la Database Diamont qui fournissent de très nombreux éléments statistiques sur l'Arc Alpin (dans le périmètre de la Conı ention Alpine), mais ils ne prennent pas en considération le franchissement des frontières.

La configuration du relief et l'enclas ement consécutif contraignent le déı eloppement des centres dynamiques, susceptibles d'attirer des frontaliers. L'effet barrière de la montagne est encore fortement présent. Certes, le caractère montagnard, rural de ces espaces transfrontaliers est un facteur explicatif éı ident à cette absence de flux de frontaliers significatif, mais il n'est pas le seul car la marge est aı ant tout la conséquence d'une relation particulière que les hommes entretiennent aı ec un lieu. Ainsi le fait que la montagne ait longtemps été considérée dans de nombreux Etats européens comme une marge parce qu'elle était éloignée, peu rentable économiquement, est un élément fondamental à prendre en considération et à contrebalancer aı ec des cas comme la Suisse et l'Autriche où la montagne n'est pas perçue comme un handicap à compenser à tout prix puisqu'elle représente la quasi-totalité du territoire du pays. Dans ces "Etats montagnards ", la I ision de la montagne $\mathrm{y}$ est différente et la logique de solidarité montagne/piémont, ou ı ille/montagne a fait son chemin et semble trouı er un écho du côté des institutions européennes en particulier au Comité des Régions (Debarbieux et Rudaz, 2010). Dès l'origine du processus de coopération institutionnelle, une I ingtaine d'années seulement pour les coopérations montagnardes, ces thématiques sont principalement orientées autour de

${ }^{4}$ http://obserı atoire.espace-mont-blanc.com/ 
l'enı ironnement, de l'agriculture de montagne et des questions culturelles (Fourny et Criı elli, 2003) mais on note depuis ces dernières années une tendance au déı eloppement de projets dans les domaines socio-économiques (dél eloppement économique, touristique, emploi, formation...) sous l'impulsion des programmes européens de coopération transfrontalière (INTERREG) et surtout durant ces quatre dernières années de la politique européenne de cohésion territoriale.

\section{Sortir de la marginalité dans un contexte d'entre-deux}

L'Union Européenne offre aux territoires périphériques et spécifiques une opportunité d'intégration par la prise en considération de leurs spécificités. Elle a déı eloppé depuis sa création une politique régionale et mis en œuı re depuis 1988 une politique de cohésion. Initialement, la ı ocation de la politique régionale était de préparer l'élargissement de I'Union à de nouı eaux membres. Aujourd'hui, elle se met au serı ice de la politique $d^{\prime}$ 'inno ation. Cette dernière est aı ant tout une politique d'inı estissement, dont l'objectif est de soutenir la création d'emplois, la compétitiı ité et la croissance économique, l'amélioration de la qualité de I ie et le déı eloppement durable. La politique de cohésion n'a pas ı ocation à contrebalancer les effets potentiellement négatifs des politiques de compétitiı ité et de concentration sur les territoires d'excellence. En rel anche, elle doit permettre à chaque région de déı elopper sa créatiı ité en mettant en aı ant ses spécificités territoriales. Elle a aussi pour objectif d'encourager la liberté d'entreprendre au niı eau régional en allant dans le sens du renforcement de la démocratie participatiı e locale. En effet, la mise en place d'une gouı ernance locale adaptée est un bon moyen de faire remonter des idées originales pro enant directement du territoire, assurant aussi une meilleure cohérence globale sur le territoire.

La politique de cohésion territoriale, dernière née des politiques de cohésion, a pour objectif de faı oriser un déı eloppement plus équilibré en réduisant les disparités existantes et en éı itant les déséquilibres territoriaux. L'article 174 du traité sur le fonctionnement de l'Union Européenne, tel que modifié par le traité de Lisbonne, accorde une attention particulière " aux zones rurales, aux zones où s'opère une transition industrielle et aux régions qui souffrent de handicaps naturels ou démographiques graves et permanents telles que les régions les plus septentrionales à très faible densité de population et les régions insulaires, transfrontalières et de montagne ". Ainsi nos territoires d'entre-deux montagnards et transfrontaliers sont doublement concernés par ces politiques.

Enfin, le cinquième rapport de la Commission européenne sur la cohésion de 2010 précédemment cité place l'inno ation au cœur de la politique de cohésion et rappelle qu'elle est le moteur principal du déı eloppement régional. II met aussi en aı ant la capacité des institutions à organiser, déı elopper, promouı oir les innoı ations sur leur territoire. Au premier abord, il est loisible de s'interroger sur la compatibilité entre les deux concepts de compétitiı ité territoriale et de cohésion. La ı olonté de l'Union n'est bien éı idemment pas de mettre ses territoires en compétition mais bel et bien de renforcer sa compétitiı ité globale à l'échelle communautaire par un processus de territorialisation de la compétitiı ité. Dans cette optique, comment s'inscrit "l'innol ation d'entre-deux » dans ce contexte de compétitiı ité ?

Montagne et frontière sont très souı ent perçues comme une barrière qui sépare, différencie les populations de part et d'autre. Le relief ajoute de la séparation par la distance sans pour autant différencier. Les concepts de limilalité/liminarité et d'hybridité permettent 
d'expliquer et de dépasser cette barrière et d'aller ı ers la notion de carrefour, qui, elle, rassemble et rapproche.

\section{La montagne frontalière : espace liminaire et lieu d'hybridité}

La frontière comme limite fonde la notion d'entre-deux, la montagne frontalière peut aussi être appréhendée sous le prisme de la liminalité ou liminarité.

\section{L'apport de l'anthropologie à l'étude des espaces d'entre-deux}

La liminalité ou liminarité ${ }^{5}$ est un concept utilisé pour étudier les situations d'entre-deux auxquelles les indiı idus peuı ent être confrontés au cours de leur ı ie. Ce concept anthropologique inı enté par Arnold Van Gennep (Van Gennep, 1909) au début du XXe siècle et repris par Victor W. Turner (Turner, 1969) pour décrire les rites de passage, dépasse la notion de limite. II sous-tend une transformation, un dépassement de la norme. Il englobe la notion d'entre-deux, à la fois spatial et temporel. C'est pourquoi ce concept nous semble particulièrement intéressant pour aborder à la fois la thématique de la frontière et celle de la montagne. La marginalité spatiale telle que nous l'aı ons décrite est une forme d'entredeux, donc de liminalité. La dimension temporelle est fondamentale pour notre étude et est étudiée par la liminalité. L'état de liminalité n'a normalement pas ı ocation à durer mais la notion peut aussi être utilisée pour décrire une condition durable comme la situation de handicap ou encore la situation d'exclusion des personnes sans domicile fixe, qui ne sortent de leur condition qu'au moment de leur mort pour les premières ou de leur réinsertion dans la société pour les secondes ou de leur disparition. En géographie, la liminalité caractérise une étendue de passage et de transition, on peut citer par exemple les objets géographiques tels que les confins, les lisières, les friches, les espaces périurbains... "L'espace liminal est celui où se gère la relation et où se fixe le statut social de celle-ci. La liminalité permet de ce fait de saisir cette dynamique entre la forme spatiale de la frontière et la fonction frontalière de l'espace " (Fourny, 2014).

Les territoires de montagne se trouı ent confrontés à de nouı eaux enjeux, notamment liés au changement climatique qui impactent directement leur économie lorsqu'elle repose en grande partie sur l'or blanc. Les territoires supports de stations de ski doil ent inno er en matière d'offre touristique ; diı ersifier leurs actiı ités afin de proposer un plus large panel mieux réparties au cours de l'année. D'autres territoires sont confrontés à l'installation massiı e de nouı eaux habitants, induisant de nou elles questions car ces nou eaux résidents présentent des caractéristiques spécifiques très différentes des " habitants traditionnels ". Ils résident dans des communes de montagne mais n'y traı aillent sour ent pas. Une adaptation du territoire est aussi nécessaire pour répondre à ces pratiques. Comme nous l'aı ons dit précédemment, ces territoires doiı ent aussi trouı er leur place dans une Europe de la cohésion qui promeut la compétitiı ité et fait de l'innol ation le moteur du déı eloppement.

${ }^{5}$ La problématique de la liminalité dans l'analyse des frontières en montagne est actuellement l'objet d'un axe de tral ail du labex ITEM (programme "Investissements $d^{\prime}$ avenir » Labex ITEM- ANR-10-LABX-50-01) 
La géographie et l'anthropologie se réı èlent complémentaires dans leur approche d'un même espace ı écu, approprié. Montagne et frontière, en tant que limite sont des points de rencontre aı ec l'altérité. L'anthropologie apporte sa contribution dans l'explication du processus géographique de recomposition territoriale à l'œu re autour de la frontière, en interrogeant ce rapport particulier à l'altérité mais aussi à la norme en prenant en considération la temporalité. Ce concept permet aussi d'étudier la frontière dans son rapport entre l'espace et la norme. La norme s'exprime sur l'espace, qui est lui-même le lieu de construction des relations. Dans le cas de la coopération transfrontalière Alpi-Marittime Mercantour, la présence de la frontière est encore très marquée, notamment parce qu'elle délimite des territoires étatiques marqués par une identité nationale forte allant jusqu'à une confrontation identitaire (Bergamaschi, 2012). L'identité culturelle et la langue constituent une autre différence majeure. Du coté de l'Espace Mont-blanc, l'usage de la langue française issue du franco-proı ençal est reı endiqué dans la Vallée D'Aoste et est reconnue comme langue officielle dans le Valais. Cependant, la population ne semble pas aı oir conscience de ı iı re dans un territoire commun transfrontalier. L'espace frontalier d'entre-deux constitue une opportunité pour tisser des liens, c'est le lieu où se créent les opportunités. Celles-ci concernent différents domaines : économiques, culturels, enı ironnementaux. La rencontre de l'altérité est certainement plus flagrante dans les relations qui se lient autour des projets culturels, aı ec comme objectif sous-jacent le maintien des traditions locales, mais aussi l'échange et le partage des spécificités. Ainsi, les combats de I aches ont repris de l'ampleur aı ec le soutien de l'Espace Mont-Blanc aux éleı eurs, qui organisent des combats transfrontaliers et décernent, depuis 2010, le titre de Reine de l'Espace Mont-Blanc.

Les frontières ont longtemps séparé les populations qui I iı aient de part et d'autre, mais leur ouı erture a renforcé les contacts qui existaient historiquement. Les premières politiques de cohésion se sont d'ailleurs appuyées sur la culture commune des populations alpines. Les différences profondément ancrées tendent à s'harmoniser du fait des échanges, les uns apprenant des autres, aı ant de mettre en commun et d'expérimenter ensemble. C'est en cela, que les espaces autour des frontières sont par excellence des lieux d'entre-deux, où l'hybridation se fait presque naturellement. Elle peut être encouragée par des échanges institutionnels, mais à l'origine elle est spontanée. Des solutions inno antes sont éprou ées localement et peuı ent être exportées, à condition d'être relayées par les acteurs et les institutions territoriales.

\section{Montagne et hybridité}

La montagne présente intrinsèquement des caractéristiques hybrides, qui renforcent la qualification d'entre-deux que nous posons sur la montagne frontalière.

On a souı ent en tête l'image d'une montagne rurale éloignée des centres urbains, ce qui n'est pas totalement । rai. Si les । illages et hameaux prédominent, ils côtoient les । illes, petites et moyennes, dont les préoccupations urbaines sont similaires aux métropoles périalpines. Les préoccupations des communes rurales et urbaines ne sont pas opposées mais se complètent, car il y a une I éritable interdépendance entre elles. II existe aussi une montagne qui emprunte des éléments au rural et à l'urbain, aı ec des enjeux économiques à la fois agricoles et touristiques. C'est le cas, par exemple, des I allées qui possèdent des stations de sport d'hiı er, urbanisées et très fréquentées une partie de l'année. Ces lieux hybrides doil ent composer al ec des infrastructures surdimensionnées, des flux de 
populations saisonnières, une économie de plus en plus diı ersifiée, et une saison morte où la collectiı ité doit être en mesure de maintenir des serı ices pour les résidents permanents. La confrontation entre ces " deux mondes " n'est sans doute pas aussi présente que dans le cas du périurbain, tiers-espace hybride par excellence (Vanier, 2000), mais on note une complémentarité ı oire une dépendance entre le monde agro-pastoral et l'industrie du tourisme qui se dél eloppe dans certaines I allées de montagne. Le maintien d'une agriculture de montagne, notamment de l'agro-sylı o-pastoralisme, est une nécessité pour conserı er un paysage ouı ert qui fait la réputation des ı allées alpines, permettant aussi d'entretenir les pentes et de réduire le risque d'aı alanche. Trouı er un équilibre entre l'urbanisation, le déı eloppement d'actiı ités économiques consommatrices d'espace et le maintien d'une actiı ité agricole traditionnelle est très délicat et impose parfois aux deux parties de s'adapter et de proposer des solutions nouı elles.

Enfin, la montagne occupe un rôle stratégique par rapport aux centres métropolitains de piémont, qui se manifeste par un rapport de dépendance dans différents domaines comme l'énergie, la production agricole ou encore les aménités. La CIPRA ${ }^{6}$ a démontré, dans son troisième rapport de 2007, le lien de dépendance énergétique des centres urbains situés autour des Alpes par rapport aux ı allées productrices d'hydroélectricité. Les I allées de montagne, en particulier celles localisées à proximité des I illes, participent à l'équilibre des centres, dans la mesure où on obserı e des migrations pendulaires de traı ailleurs ou encore des flux réguliers notamment pour les loisirs le week-end. En outre, la montagne occupe un rôle stratégique en tant que système d'alerte précoce dans le contexte actuel de changement climatique, les I ariations de température étant plus marquées.

Les I illes intra-alpines retrouı ent toute leur importance et déı eloppent des réseaux entre elles mais aussi aı ec les ı illes périalpines. Ce rapport de dépendance des métropoles par rapport aux Alpes, associé à la localisation centrale des Alpes au sein de l'Europe, en fait un ı éritable carrefour. Ceci nous incite à dire que le rapport centre-périphérie classique deı rait être rediscuté, enı isagé d'une nouı elle manière. Dans une perspectiı e plus globale d'inı ersion du rapport centre-périphérie, les Alpes deı iendraient le centre de l'Europe, et les I illes autour constitueraient la périphérie. Les Alpes constituent déjà un centre important, c'est depuis ce centre que l'Union Européenne s'est élargie à l'Est et c'est aussi par ce carrefour que transitent les biens et marchandises à traı ers l'Europe. Le but n'est pas d'ignorer les I illes périalpines ou de contester leur effet polarisant. Au contraire, il s'agit de mettre en aı ant les relations I illes-montagnes depuis la montagne. A défaut de se défaire de l'approche traditionnelle centre-périphérie, l'Union européenne a opté pour une approche fonctionnelle, multiscalaire des territoires.

Le Schéma de Déı eloppement de I'Espace Communautaire (SDEC), adopté en 1999 dans le cadre de la politique régionale européenne, est un document d'orientation de la politique territoriale et spatiale de I'Union. II encourage le polycentrisme à toutes les échelles $d^{\prime}$ interı ention : mondiale et continentale, nationale et transnationale, régionale et locale. Dans le cadre des coopérations transfrontalières montagnardes, le dés eloppement de relations aı ec les métropoles régionales est indispensable à l'échelle des coopérations interrégionales pour déı elopper des stratégies territoriales communes. A l'échelle des

6 CIPRA: Commission Internationale pour la Protection des Alpes http://www.cipra.org/fr/CIPRA/cipra-france 
coopérations locales, le polycentrisme se manifeste par le dél eloppement de relations étroites entre les I allées montagnardes et les I illes de piémont, elles-mêmes connectées aux métropoles régionales. Ces relations ont pour but de créer de I éritables solidarités I allées montagnardes/ı illes de piémont. Solidarité ne signifie pas dépendance, mais plutôt mettre l'accent sur les complémentarités.

Dans un contexte frontalier, le ou les centres urbains les plus proches en distance ou en temps de parcours ne correspondent pas toujours aux centres régionaux de l'Etat de résidence. Si pour le commerce cela ne pose pas de problème majeur, en reı anche lorsqu'il s'agit des serı ices administratifs ou de santé, les choses se compliquent. Se faire soigner dans un Etat I oisin complique le remboursement.

\section{Les Alpes, carrefour de l'Europe}

Les Alpes sont aussi un carrefour de l'Europe, qu'il faut tral erser par des cols historiques ou des tunnels, se situant très sour ent sur des frontières (tunnels du Mont-Blanc et Fréjus entre France et Italie, col du Grand Saint-Bernard entre Italie et Suisse). Ce massif a joué un rôle central dans la construction européenne et notamment son extension à l'Est. Le transport est un facteur majeur pour la cohésion et le déı eloppement économique et est l'objet d'enjeux et de tensions importantes.

Si la montagne perçue comme une barrière aux relations sociales et commerciales subsiste encore dans l'esprit de certaines personnes, en particulier des non montagnards, comme une limite aussi forte que la frontière, elle a été aı ant tout un élément rassembleur des populations alpines, un lien culturel fort qui a permis de dépasser la diı ersité linguistique. Gian Paolo Torricelli a montré comment au XVIIle siècle, l'édification " de routes à col " a été un moyen pour les Etats d'affirmer leur puissance territoriale et de maîtriser leurs frontières, aı ec pour conséquence le déclin des petites i illes alpines. Pourtant les cols, plus que des frontières sont, depuis le Moyen-Age, des traits d'union entre deux I allées parfois de nationalité différente. Si la mobilité en montagne est contrainte, les populations alpines ont toujours circulé et I aincu l'isolement lié à l'enclaı ement des I allées. Les Alpes sont de par leur situation centrale en Europe, un carrefour et ne constituent pas un obstacle, à peine un ralentissement (Torricelli, 2002). Elles sont le lien entre les métropoles, les axes de communication les traı ersant jouant un rôle fondamental pour le déı eloppement à la fois des Alpes mais aussi des régions périalpines. Soulignons les efforts des Etats Alpins pour améliorer la circulation et la traı ersée des Alpes, par la construction, la réno ation d'infrastructures d'enı ergure (tunnels routiers ou ferroı iaires, autoroutes), ce qui contribue à l'attractiı ité de ces régions. Le déı eloppement des moyens de communication associé à la circulation des modèles et représentations contribue à modifier profondément les territorialités frontalières mais aussi les territorialités à l'échelle des Alpes. Les I illes moyennes alpines sont de plus en plus reliées entre elles et reliées aı ec les I illes de piémont et même au sein d'un système de i illes européennes (Perlik, 1999). Elles déı eloppent des réseaux interconnectés dépassant les frontières nationales. Ces interconnexions permettent de repenser les relations transfrontalières. La fermeture du tunnel du Mont-Blanc suite au dramatique incendie de 1999 pendant trois ans a eu comme impact direct le report des flux sur le tunnel du Fréjus et de nombreux impacts indirects sur l'économie locale des deux I allées frontalières. La fermeture progressiı e de l'hôpital de Chamonix, d'abord la maternité, puis le bloc opératoire et enfin les urgences en dehors des saisons touristiques d'été et $d^{\prime}$ 'hil er est le fruit d'une politique de rationalisation française qui n'a pas pris en compte les interconnexions possibles et les possibilités d'accords aı ec la I allée d’Aoste. Dans le 
domaine de la santé toujours, une réflexion est en cours aı ec les autorités italiennes, en particulier la ı ille de Turin, pour maintenir le serı ice de réanimation de l'hôpital de Briançon, fondamental en zone de montagne.

\section{La montagne frontalière : laboratoire d'innovation à la marge}

Certains auteurs mettent en aı ant le fait qu'il serait plus facile d'inno er à la marge, car elles seraient porteuses d'une moindre charge symbolique et politique. Cependant il ne faut pas surestimer leurs capacités à inı enter ou créer de nouı eaux modèles spatiaux (Antheaume et Giraut, 2002). Cette affirmation est ı raie mais à nuancer pour les montagnes, qui sont dotées d'une symbolique forte dans l'esprit des populations, notamment urbaines : " la montagne jardin ou encore terrain de jeux ". De plus, les élus de la montagne ont un poids assez considérable à l'échelle nationale, en particulier en France.

En second argument, la marge serait un élément fort d'identification territoriale positiı e susceptible de faire émerger l'inno ation. En effet, la capacité d'innol er des communautés alpines, et plus généralement des communautés de montagne, tient à leur inclusion dans un système de contraintes et de potentiels. L'adaptation aux conditions particulières (climat, pente, isolement lié à l'enclaı ement) a été la première source d'inno ation. Aujourd'hui encore, c'est l'éı olution des conditions enı ironnementales mais aussi économiques qui contraignent les populations à trouı er de nouı elles solutions pour répondre à leurs besoins et aux nour elles problématiques.

Autre argument, c'est dans les situations bloquées, inédites, ou spécifiques, que le besoin $d^{\prime}$ inno ation serait le plus fort. La confrontation I oire la contradiction de plusieurs logiques : celle d'en haut issue des instances européennes (procédure top down) et celle du bas (procédure bottom-up) issue de l'échelon local peut conduire à des processus inno ants, notamment en matière de gou ernance et d'action publique.

Pour finir, le terme " innol ation » ne signifie pas nouı eauté, ou inı ention. L'inı ention n'est qu'une potentialité alors que l'inno ation consiste en l'implantation effectil e et durable d'une inı ention dans un milieu social (Alter, 2013). La nouı eauté peut être d'ordre organisationnelle, le processus d'inno ation correspond à l'appropriation et à la diffusion de cette nouı eauté dans le milieu social. II peut s'agir aussi de réinı ention, de bricolage entre du " I ieux " et du " neuf ", d'un assemblage complexe entre des procédures et processus communs et d'autres nouı eaux inı entés localement ou reçus par diffusion depuis le centre. On reste donc dans le champ de l'entre-deux. Dans notre cas, l'inı ention est un outil juridique de l'Union Européenne (GECT), proposant un modèle de goul ernance. Les coopérations transfrontalières montagnardes s'en emparent progressil ement comme l'ont fait précédemment les grandes régions transfrontalières, de la même façon qu'elles se sont appropriées les différents outils européens (programmes Interreg).

\section{Montagne : de la marginalité à la reconnaissance européenne}

Pendant plusieurs décennies, la montagne a été le support d'une marginalité politique, qui tend aujourd'hui à être effacée par des politiques I olontaristes, nationales et européennes. Cependant, si la ı olonté de désenclaı er et surtout de dynamiser ces territoires est bien présente, le modèle d'action ne semble pas toujours approprié, ce qui impose ou permet, selon le point de ı ue, d'inno er dans la mise en œuı re de nouı elles politiques publiques. 
Dans le même temps, les politiques sectorielles ont fait l'objet d'adaptation aux spécificités des régions de montagne ou à celles des régions frontalières. Mais depuis le Traité de Lisbonne signé en 2007, on note un net changement au moins dans les textes, issu d'une I olonté d'améliorer l'efficacité des politiques en assurant une meilleure coordination entre elles et, surtout, en menant des politiques transı ersales incluant la politique de cohésion territoriale. La transı ersalité est fondamentale, en particulier dans ces territoires périphériques éloignés des centres, où chaque interı ention par le biais d'une politique dédiée impacte un autre domaine. Prenons un exemple concret, celui de la mise en place d'une action en matière de serı ice à la personne. II faut pour cela recruter du personnel qualifié, cela concerne donc le domaine de l'emploi, mais aussi celui de la formation. Ces questions sont importantes car les centres de formation se trouı ent rarement en montagne, obligeant les acteurs à créer et entretenir des partenariats aı ec les centres urbains de proximité mais aussi les ı illes alpines disposant d'uniı ersités et de centres de formation professionnelle. Ces personnes I ont de oir circuler pour se rendre sur les lieux de formation, ce qui implique de prés oir un réseau de transport multimodal. Cela interroge aussi le domaine des TIC (Technologies de l'information et de la communication) qui I ont permettre une formation à distance, à condition de déı elopper des réseaux suffisamment performants. L'inno ation résulte ici du traitement global d'une problématique, par la mise en œuı re de solutions transı ersales.

\section{Innovations transfrontalières et outils juridiques}

Les programmes européens INTERREG créés en 1990, notamment pour promour oir la cohésion et le dél eloppement des espaces frontaliers sont rapidement deı enus l'outil incontournable de financement des projets transfrontaliers.

Ces dernières années, le Parlement et le Comité des Régions encouragent très fortement les coopérations locales et territoriales à s'institutionnaliser, en utilisant leur outil juridique phare, à saı oir le Groupement Européen de Coopération Territoriale (GECT), en en faisant pour la prochaine programmation européenne 2014-2020, l'autorité de gestion des fonds européens. Ceci représente un pas important de la part de l'Union, qui souhaite ı oir les actions transfrontalières se pérenniser au delà du cycle de I ie des projets. L'objectif second est d'éı iter la logique de guichet qui prédominait jusqu'alors et qui contribuait à encourager les financements dits d'opportunité. Le GECT issu du règlement européen No 1082/2006 du 5 juillet 2006, a fait l'objet d'un projet de règlement modificatif le 6 octobre 2011 de la part de la Commission européenne, le but étant de promouı oir son utilisation et d'assouplir les conditions de création.

Comme tout outil juridique, le GECT implique la mise en place d'une gou ernance multiniı eaux qui garantie l'implication des différents échelons territoriaux (du local au national en passant par le niı eau régional). Ce cadre, en apparence relatiı ement souple, se réı èle pour des territoires peu institutionnalisés compliqué à mettre en œuı re. En effet, la gestion quotidienne n'est que très peu formalisée et est très modulable par rapport au cadre imposé par le règlement européen. Cette gouı ernance locale doit aussi permettre d'inclure les représentants de la société ciı ile afin d'alimenter le processus d'inno ation par le biais de la démocratie participatiı e.

L'Union Européenne définit une politique, impulse un mour ement, propose des outils et finance la mise en œu re des politiques sur les territoires, à charge pour ces derniers, de s'adapter pour répondre aux orientations européennes. Ce processus d'appropriation de la nou eauté I a conduire à une inno ation si l'appropriation par les acteurs est positiı e, dans 
le cas contraire la nou eauté sera considérée comme un échec (Alter 2013). La réception de la nouı eauté n'est pas uniforme selon les milieux et peut être plus ou moins longue et se faire selon des modalités différentes.

L'outil GECT a été rapidement approprié par les coopérations métropolitaines, ou les coopérations eurorégionales ou macrorégionales, mais plus difficilement à l'échelon local. Dans les Pyrénées, deux GECT se sont créés: le premier, en 2010 le GECT Hôpital de Cerdagne dont la I ocation est de garantir une offre de soins transfrontalière sur un I aste périmètre (53 communes) ; le second en 2011 GECT « Espace Pourtalet " a comme mission principale l'amélioration de l'entretien de la ı oie transpyrénéenne qui traı erse le col du Pourtalet à 1795 mètres d'altitude. Dans les Alpes occidentales, le premier GECT a I u le jour le 6 juin 2013, il s'agit aussi du premier parc européen, qui unit après 26 ans de jumelage le Parc national du Mercantour (France) et le Parco Naturale delle Alpi Marittime (Italie). Le nombre de GECT créés en montagne est faible. Nous pouı ons aı ancer comme premier élément d'explication, que le GECT n'a pas été conçu uniquement, comme son nom l'indique, pour les coopérations transfrontalières, mais pour les coopérations territoriales, ce qui change clairement la donne. En effet, la nature des coopérations territoriales n'est pas exactement la même, tout comme l'échelle de coopération. Les enjeux ne sont pas identiques au niı eau local et au niı eau régional, c'est pourquoi l'analyse multiscalaire est fondamentale pour répondre aux questions suil antes : la délimitation d'un espace de coopération sous-jacente au processus d'institutionnalisation ne contribue-t-elle pas à le faire sortir de sa position marginale ? Ne repousse-t-on pas les marges à la périphérie du territoire de coopération?

Délimiter précisément un périmètre de coopération reı ient à fixer de façon plus ou moins définitiı e le territoire d'une coopération transfrontalière, car toute modification, en particulier des membres (commune ou regroupement de communes), implique de reprendre à zéro le processus de création du GECT. En France, pour l'instant aucune commune n'est membre d'un GECT, ce sont les Etablissements Publics de Coopération Intercommunale (EPCI) qui adhèrent. L'action d'un GECT peut s'étendre sur le territoire d'une commune non membre, mais cela peut poser à terme des problèmes de représentation et de goul ernance, car les communes non membres ne sont pas représentées dans les instances de gou ernance. Cette rigidité ne correspond pas I raiment à la réalité matérielle des coopérations montagnardes qui sont mouı antes. Les communes participent au gré de leurs intérêts, leur implication peut donc être fluctuante. De plus, le périmètre de coopération de rait refléter un territoire de coopération sur lequel s'inscrit un projet. Or le projet de territoire n'est pas toujours très clairement défini, les grandes orientations autour d'un axe central, souı ent le dél eloppement durable, sont présentes mais les relations aı ec les acteurs restent à déı elopper.

La critique principale que nous pouı ons faire à la politique de cohésion territoriale de I'Union concerne son modèle. En effet, elle a été construite sur le modèle dominant des métropoles mais se ı eut applicable à toutes les régions et en particulier aux régions les plus défaı orisées, qui ne sont pas pour la majorité des métropoles. Cela ne ı eut pour autant pas dire que ces espaces ne peuı ent pas s'approprier les outils tels que le GECT. Ils doil ent simplement s'adapter en trouı ant des "solutions inno antes " c'est-à-dire acceptées politiquement et socialement, ce qui requiert du temps. L'adaptation semble plus simple et moins coûteuse en temps lorsque les coopérations transfrontalières ou régionales sont déjà 
aı ancées dans leur processus d'institutionnalisation car des habitudes de collaboration sont déjà en place. Les acteurs des territoires se connaissent et collaborent déjà, les chargés de missions ont une certaine expérience des programmes opérationnels. En reı anche, le coût d'entrée pour les communes qui ne font pas partie de la coopération institutionnelle est nettement plus éleı é, la solution pour elles étant de se raccrocher au processus en deı enant membre d'une coopération transfrontalière ı oisine en cours d'institutionnalisation.

Ces coopérations transfrontalières montagnardes européennes (et alpines pour les cas étudiés) sont caractérisées par une gouı ernance multi-niı eaux comme les coopérations transfrontalières de type métropolitaines mais on note un fort degré d'informel, c'est-à-dire que la coopération est daı antage basée sur des relations interpersonnelles de type informel que sur des relations institutionnelles. L'informel a très largement progressé au cours des cinq dernières années, notamment aı ec la réalisation de Plans Intégrés Transfrontaliers $(\mathrm{PIT})^{7}$. Concrètement les grands rendez-ı ous transfrontaliers (par exemple Conférence Transfrontalière Mont-Blanc) ont été remplacés par des réunions plus régulières entre les différents acteurs du transfrontalier et par des contacts informels (mail, téléphone, I isioconférence). En outre, de nouı elles formes d'action collectiı e émergent, prennent de l'ampleur et peui ent s'inscrire dans la durée lorsqu'elles sont relayées par les acteurs institutionnels des territoires concernés. Les relations informelles constituent une source priı ilégiée pour le déı eloppement des réseaux sur les territoires spécifiques d'entre-deux car elles n'obéissent à aucune règle formelle, elles s'organisent au gré des liens qui se tissent au quotidien entre les acteurs. De plus, l'accroissement des relations informelles n'est pas contradictoire aı ec la mise en œuı re d'une gouı ernance formelle institutionnalisée. Au contraire, c'est un bon préalable qui offre la possibilité d'expérimenter de nour elles méthodes de communication, de participation de la société ciı ile à la i ie locale transfrontalière. Les relations informelles peuı ent être encouragées par les institutions ou par les autorités administratil es qui peuı ent offrir les conditions de leur éclosion en faı orisant les rencontres par la mise à disposition de lieux d'échange, de rencontre, mais aussi en préı oyant des projets transfrontaliers impliquant les populations, toutes les catégories de populations. En effet, les projets I isent souı ent une catégorie particulière de la population, en particulier les jeunes d'âge scolaire.

La singularité montagnarde est plus qu'affichée dans les régions transfrontalières alpines étudiées, elle est même reı endiquée comme facteur d'innol ation par les acteurs locaux. L'objectif est de répondre, par des solutions locales, à des enjeux locaux. L'exemple de la coopération entre les deux parcs Alpi-Marittime et Mercantour est assez emblématique. Bien que les objectifs soient communs, à sal oir la protection des milieux et le déı eloppement local, les moyens diı ergent tant du point de I ue de leur conception que de leur application. Cependant, la ı olonté commune affichée depuis près de 25 ans de faire ensemble, leur permet progressil ement d'étendre le champ de leurs actions communes. De nombreuses barrières ont été dépassées, celles de la langue, des cultures politiques et administratiı es pour tenter de porter ensemble une I ision commune d'un territoire qui a I ocation à s'agrandir. De son côté, l'Espace Mont-Blanc correspond en tout point à la ı ision

\footnotetext{
${ }^{7}$ Les Plans Intégrés Transfrontaliers sont les outils de réalisation des programmes ALCOTRA 2007-2013 (INTERREG France-Italie)
} 
de la montagne frontalière à la fois barrière et carrefour que nous aı ons tenté de décrire ; son logo représentant trois hommes unis autour d'une même montagne l'explicite parfaitement.

\section{Bibliographie : (à revoir selon la feuille de style)}

Alter N. (2013) L'innol ation ordinaire, Paris, PUF, 392p.

Amilhat-Szary A-L., Fourny M-C (2006) Après les frontières, aı ec la frontière: Nouı elles dynamiques transfrontalières en Europe, Editions de l'Aube, $169 \mathrm{p}$.

Antheaume B., Giraut F. (2002) "Les marges au coeur de l'innol ation territoriale? Regards croisés sur les confins administratifs (Afrique du Sud, France, Maroc, Niger, Togo ... .) ", Hist. Géographes, $n^{\circ} 379$, p. 39-58.

Bart F. (2001), "Montagnes entre marginalité et intégration ", in Veyret V.Les Montagnes : Discours et enjeux géographiques, Sedes/VUEF, p. 51-62.

Fourny M-C. (à paraître en 2014), "La frontière comme espace liminal. Proposition pour analyser l'émergence d'une figure de la frontière mobile dans le contexte alpin », Journal of Alpine Research / Reı ue de géographie alpine.

Bergamaschi A. (2012) « Un imaginaire montagnard traı ersé par la frontière. Le Parc National du Mercantour et le Parco Alpi Marittime. ", Migr. Société, 2012, I ol. 24, n¹40, p. 243-254.

Broggio C. (2002) « La politique de la montagne en France », Hérodote, 2002, I ol. №107, n4, p. 147-158.

Brunet R., Ferras R., Théry H. (1993) Les mots de la géographie: dictionnaire critique, RECLUS, $532 \mathrm{p}$.

Comte H., Le rat N. (2006) Aux coutures de l'Europe: défis et enjeux juridiques de la coopération transfrontalière, Editions L'Harmattan, $365 \mathrm{p}$.

Debarbieux B., Rudaz G. (2010) Les faiseurs de montagne: imaginaires politiques et territorialités, XVIIle-XXIe siècle, CNRS, $373 \mathrm{p}$.

Debarbieux B. (1997) «La montagne comme figure de la frontière: réflexions à partir de quelques cas. », Le Globe, 1997, I ol. TOME 137, p. 149-159.

Fourny M.-C., Criı elli R. (2003) « Cette montagne que I'on partage. Frontière et montagne dans les coopérations transfrontalières de régions alpines ". JAR-RGA 2003, n³, p. 57-70.

Giraut F. (2009) «Préface. Innol ations et territoires: Les effets contradictoires de la marginalité. ", JAR-RGA, n97-1.

Guichonnet P., Raffestin C. (1974) Géographie des frontières, Presses Unil ersitaires de France, $236 \mathrm{p}$.

Lefebı re C. (2007) « Mobilités, traces et frontières dans l'Afrique des Grands Lacs.» Afr. Riı . Trimest. Studi E Doc. Dell'Istituto Ital. L'Africa E L'Oriente, 2007, I ol. 62, n4, p. 598-605.

Perlik M. (1999) « Les Alpes, les I illes petites et moyennes et l'Europe. Les I illes des Alpes, partie intégrante d'un système de i illes européen. ", JAR-RGA 1999, । ol. 87, n², p. 9-21.

Prost B. (2004) « Marge et dynamique territoriale » Géocarrefour, I ol. 79, n², p. 175-182.

Raffestin C. (1986) Eléments pour une théorie de la frontière, 1986, < http://archil e-oul erte.unige.ch/ı ital/access/serı ices/Download/unige:4348/FULLTEXT > Raffestin C. (1974) Espace, temps et frontière, < http://archiı e-ouı erte.unige.ch/ı ital/access/serı ices/Download/unige:4296/FULLTEXT > 
Raffestin C. (1992) Autour de la fonction sociale de la frontière, < http://archive-ouverte.unige.ch/vital/access/services/Download/unige:4376/FULLTEXT > Reitel B. (2011) La frontière internationale, objet sémique, processus multidimmensionnel, interface signifiante, Working Papers, N²011-43, CEPS /INSTEAD.

Sacareau I. (2003) La montagne: une approche géographique, Ed. Belin, 287 p.

TURNER V., 1969, The ritual process. Structure and anti-structure., Aldine de Gruyter. Chicago.

Torricelli G. P. (2002) « Traversées alpines, ville et territoire : le paradoxe de la vitesse ", JARRGA 2002, vol. 90, n³, p. 25-36.

Van Gennep A. (1909) Les rites de passage. Etude systématique des rites., Paris, Picard.

Vanier M. (2000) «Qu'est-ce que le tiers espace ? Territorialités complexes et construction politique ", JAR-RGA 2000, vol. 88, n¹, p. 105-113.

Zuanon J. P., Gerbaux F., Barruet J. (1984) "La politique de la montagne: entre le changement et la continuité ? ", JAR-RGA 1984, vol. 72, n², p. 329-346. 